\title{
Intrapulmonary bronchopulmonary anastomoses in COVID-19 respiratory failure
}
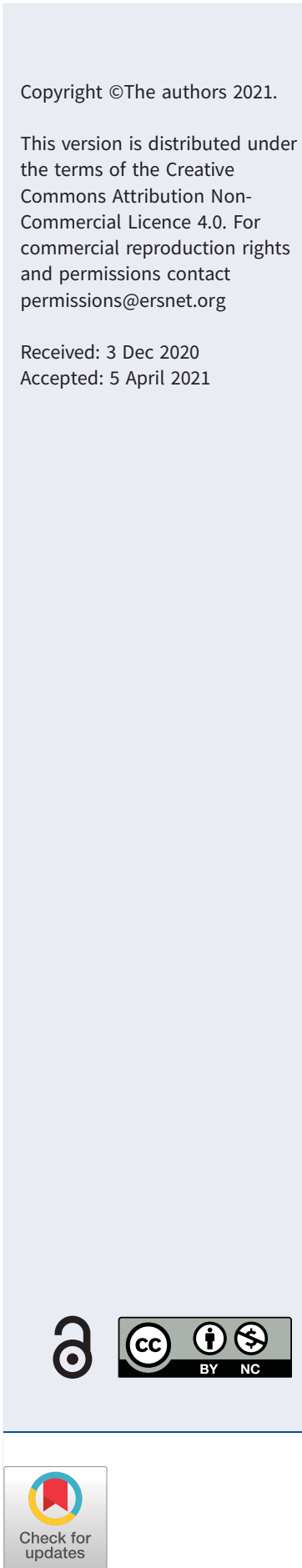

To the Editor:

The spread of severe acute respiratory syndrome coronavirus 2 (SARS-CoV-2) has led to a devastating and worldwide pandemic disease known as coronavirus disease 2019 (COVID-19). COVID-19 causes acute hypoxic respiratory failure (COVID-ARF), a major cause of mortality and morbidity, with an incompletely understood pathophysiological mechanism. GatTiNoni et al. [1] noted that COVID-19 patients with acute hypoxic respiratory failure have lung disease that is often characterised by a remarkable dissociation between relatively well-preserved lung mechanics, including lung compliance, and severe hypoxaemia. These findings are consistent with the concept that profound hypoxaemia occurring in ventilated patients with highly compliant lungs could be due to the loss of regulation of lung perfusion and impaired hypoxic pulmonary vasoconstriction. Early autopsy studies suggest that the lung circulation is a major target of coronavirus infection, which leads to striking pulmonary vascular disease due to variable degrees of thrombosis, apoptosis, oedema, inflammation and angiogenesis [2-4]. These changes contribute to dysregulation of the pulmonary vasculature, which induces perfusion abnormalities and contributes to the physiological phenotypes reported in COVID-19 pneumonia. Further, computed tomography suggests a unique "tree in bud" appearance of small pulmonary arteries [3] and transcranial agitated saline microbubble doppler studies of COVID-19 patients with hypoxaemia have demonstrated intrapulmonary shunting of these bubbles, and that the presence and degree of transpulmonary bubble transit correlates with the degree of hypoxaemia [5]. Despite these studies, histopathological correlates of severe hypoxaemia and shunt in the setting of relatively normal lung compliance in COVID-19 patients are largely lacking.

Prominent intrapulmonary bronchopulmonary anastomoses (IBA) connecting pulmonary arteries and bronchial arteries that bypass the alveoli have been characterised by three-dimensional (3D) reconstruction of tissue sections and identified as a potential source of right-to-left shunt with profound hypoxaemia in several disorders of the lungs, including idiopathic pulmonary hypertension and chronic thromboembolic pulmonary hypertension [6-13]. IBA represent pre-existing vascular connections between the bronchial and pulmonary vascular trees that are normally prominent during fetal life, appear to close at birth but can be present afterwards, especially with disease [14]. The physiological roles of these shunt vessels are incompletely understood but appear to contribute to hypoxaemia due to right-to-left shunt in response to exercise, hypoxia and catecholamine challenge [15-17]. These precapillary anastomotic connections with a diameter ranging from $15-500 \mu \mathrm{m}$ have a capability to redirect deoxygenated blood to bypass the pulmonary microvascular bed leading to poor perfusion of the distal lung [18]. Whether these vascular connections are prominent in COVID-19 lungs, reflecting recruitment of shunt vessels, has not been investigated. We hypothesised that IBA are recruited in lungs of patients who died of COVID-19 with acute respiratory failure with profound hypoxaemia.

The study was approved by the institutional review board of Icahn School of Medicine at Mount Sinai. We collected archived autopsy lung tissues from three COVID-19 patients. Haematoxylin-eosin (HE) stained slides of two tissue blocks of each patient's lung were reviewed. Routine HE sections in combination with trichrome stain and immunohistochemical stains (CD31, smooth muscle actin stains) were used to define the lung architecture and microanatomy. Our study focused on vessels in the distal lung, the sites of gas exchange, so the vessels studied were neighbouring terminal and respiratory bronchioles (with a range of airway diameter of 250-600 $\mu \mathrm{m}$ ) and in the distal airspace. The structures and pathways of IBA were

Shareable abstract (@ERSpublications)

Open intrapulmonary bronchopulmonary anastomoses (IBA) were identified in COVID-19 patients who died of respiratory failure. IBA may be the microanatomical basis of intrapulmonary right to left shunt leading to severe hypoxaemia in COVID-19. https://bit.ly/3e2GajO

Cite this article as: Galambos C, Bush D, Abman SH. Intrapulmonary bronchopulmonary anastomoses in COVID-19 respiratory failure. Eur Respir J 2021; 58: 2004397 [DOI: 10.1183/13993003.04397-2020]. 
a)
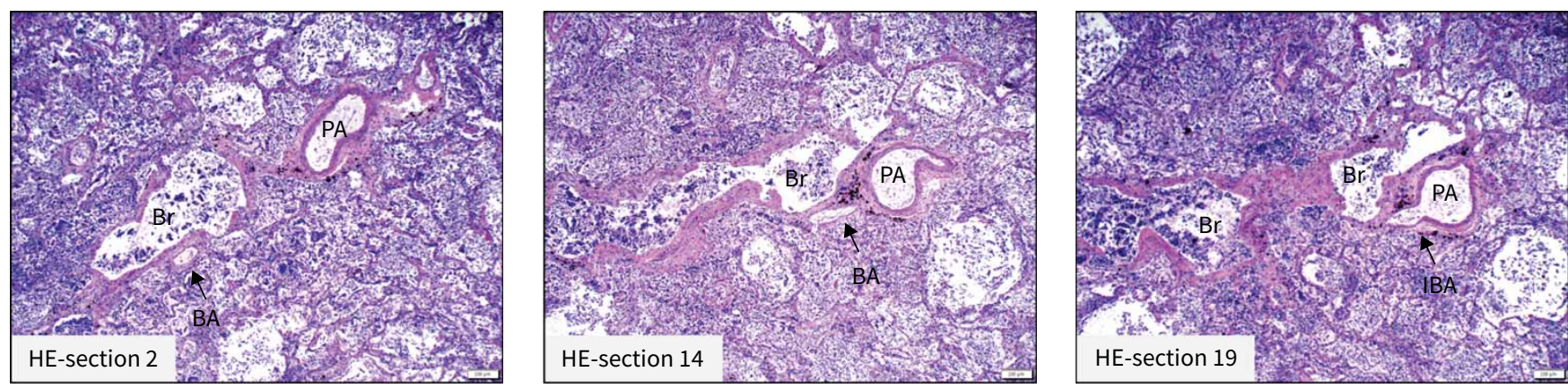

b)
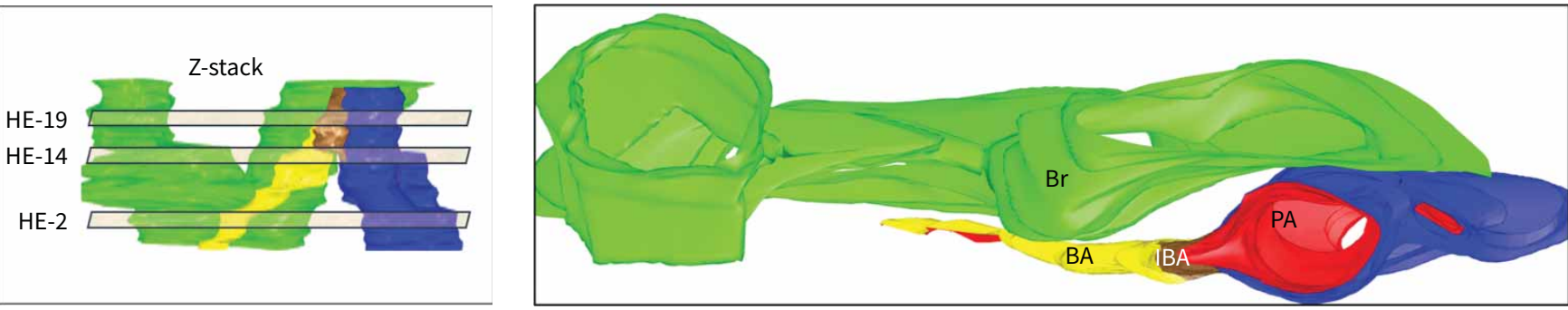

c)

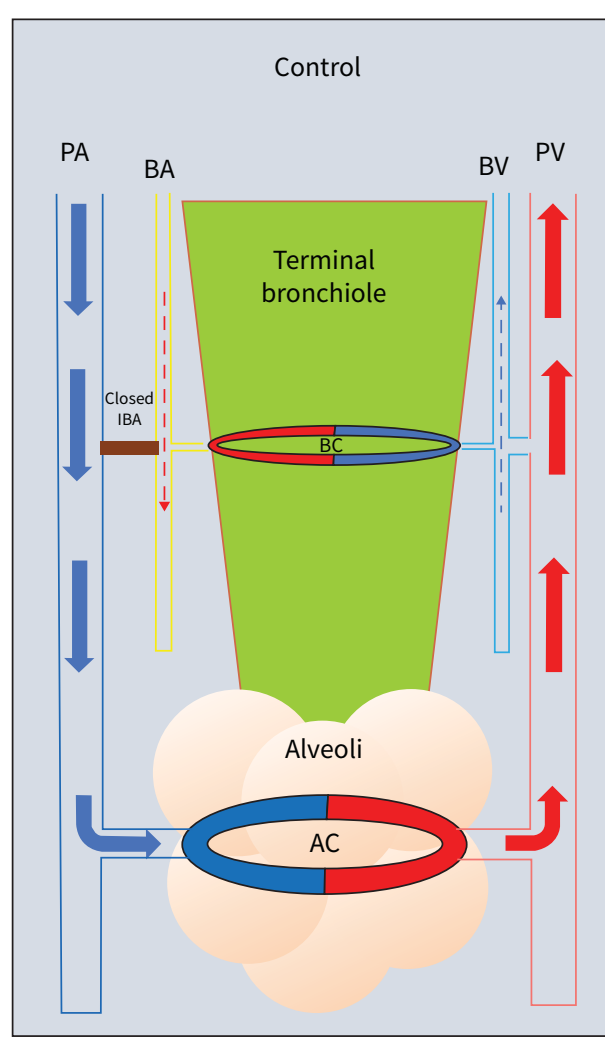

COVID-19

PA

BA

BV

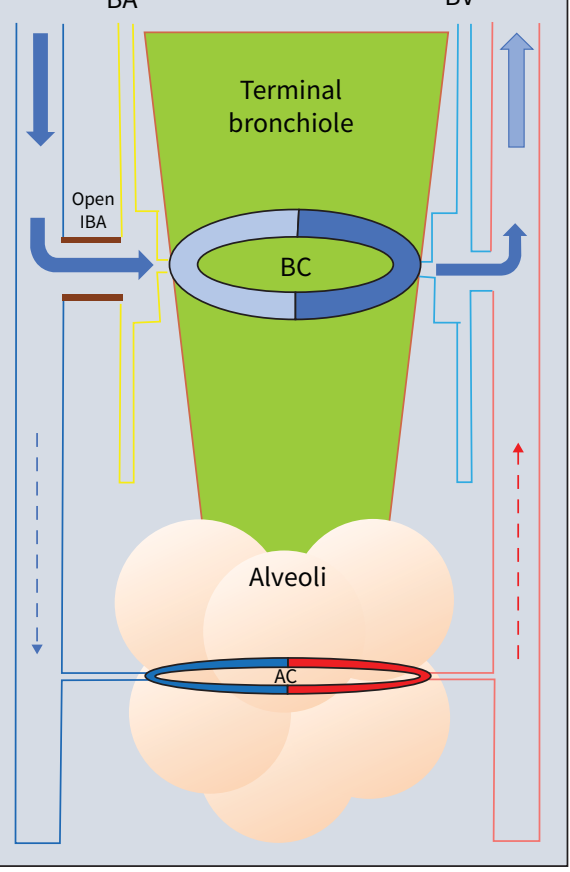

FIGURE 1 The combination of serial haematoxylin-eosin (HE) sections, and three-dimensional (3D) image reconstruction identifies recruited intrapulmonary bronchopulmonary anastomoses (IBA) in coronavirus disease 2019 (COVID-19) patients. a) Three representative images from the 30 serial HE sections analysed. Z-stack of HE sections showing an area of broncho-arterial bundle was first created (panel b, left: virtual light brown sections represent the approximate areas of the numbered $\mathrm{HE}$ sections in the $\mathrm{Z}$ stack) and a 3D image with oblique representation was reconstructed (panel b, right). There is a wide open IBA (brown in 3D image, small arrow in HE section 19) that connects the pulmonary artery (PA, blue) with the bronchial artery (BA, yellow). The bronchiole $(\mathrm{Br})$ is green and endothelium of BA, IBA and PA is highlighted by red colour. Scale bars: $200 \mu \mathrm{m}$. c) Schematic demonstrating the microanatomy of intrapulmonary right-to-left shunt. Pulmonary blood flow in the distal lung at the terminal bronchiolar level is rearranged in COVID-19 patients. In the normal lung (left panel), the deoxygenated blood (blue arrow) in the pulmonary arteries (PA) enters the alveolar capillary bed (AC) for gas exchange, and the oxygenated blood (red arrows) is collected via pulmonary veins (PV) and enters the left heart. A small amount of oxygenated blood (red dashed arrow) supplies the terminal bronchiole (green colour) via 
the bronchial artery (BA) and the bronchiolar capillary network (BC). The deoxygenated blood (dashed blue arrow) is collected by the bronchial vein (BV) and consequently the pulmonary vein that enters the left heart. The IBAs (brown colour) are closed and no blood flow between pulmonary and bronchial vascular trees is present. In the COVID-19 lung (right panel), the majority of deoxygenated blood in the distal pulmonary arteries does not reach the alveolar capillary network for gas exchange (dashed blue arrow), but is redirected (blue arrows) through open IBAs towards the bronchial arteries and bronchial microcirculation, bypassing the alveolar capillary bed. The bronchial arteries, capillaries and veins are passively dilated due to the massive amount of blood coming from the right heart. The blood remains deoxygenated and is collected by the bronchial veins and enters the left heart via pulmonary veins, contributing to the profound systemic hypoxaemia experienced by COVID-19 patients.

further studied with photographs from serial sections to create a Z-stack and then three-dimensionally reconstructed using the Free-D computer software program [19].

The demographics of the selected patients reflect a typical COVID-19 patient population with age range at the time of death of 69-86 years, male to female ratio of 2:1, common associated comorbid conditions of hypertension, diabetes mellitus and chronic kidney disease, and short hypoxaemic disease course after positive test for COVID-19 (2-10 days). All patients received COVID-19-related medication, including azithromycin and hydroxychloroquine. Chest radiographs showed patchy opacities in all patients.

Histopathological examination coupled with 3D reconstruction revealed the presence of prominent IBA in all three patients. The profiles of IBA revealed widely open anastomotic vascular connections between pulmonary and bronchial vasculature, confirmed through 3D reconstruction (figure 1). Dilated bronchial microvasculature and IBA with sizes up to $75 \mu \mathrm{m}$ in diameter were identified in all patients. We did not find histological evidence of endotheliitis, capillary microthrombi, or arterio-venous malformation. One patient had a thrombus within a distal pulmonary vein. Two patients had emphysematous changes. Histopathological changes related to acute lung injury were seen in all samples, including the variable combination of viral pneumonia, airway and interstitial inflammation, aedema, and hyalin membrane disease

Although the exact pathophysiological mechanisms underlying severe hypoxaemia in subjects with COVID-19 are uncertain, recent clinical, imaging and autopsy studies have identified abnormal pulmonary vasculature [2-4] with intrapulmonary right-to-left shunt [5] as key players in the development of silent but profound and unresponsive hypoxaemia, which leads to the significant morbidity and mortality in COVID-19 patient population. Our findings are not only in concert with the pulmonary vasculopathy paradigm developed from recent imaging studies [3, 5], but further suggest that prominent IBA may be the microanatomical correlates of COVID-19-related hypoxaemia. Using rigorous histological assessment combined with computed 3D image reconstruction, we identified prominent and recruited IBA and suggest these are potential histological correlates of intrapulmonary right-to-left shunt. Pulmonary thrombosis has been a common finding in COVID-19 patients [2-4]. Although we did not find microthrombi, we did identify a thrombosed pulmonary vein in one patient. Bronchial vessel connection to pulmonary vessels along with abnormal pulmonary veins have been described in adult patients with chronic thromboembolic pulmonary hypertension, suggesting a link between thrombotic events and the recruitment of IBA in COVID-19 patients [13]. Inflammatory and infectious airway disease has been shown to induce recruitment of IBA and it is possible that IBAs are present in patients with COVID-19 respiratory failure and hypoxaemia [20, 21].

We propose that the recruitment of IBAs and the dilated bronchial microvasculature are potential sites through which deoxygenated blood travels from right-to-left, bypassing the alveolar capillary network and impairing gas exchange (figure 1). Our findings support the recent agitated saline ultrasound study that suggests intrapulmonary right-to-left shunts as a pathological explanation for the profound hypoxaemia in COVID-19 patients [5]. This initial hypoxaemia with obstruction and poor perfusion of the distal capillary bed may be worsened by IBA recruitment, which gives rise to a profound right-to-left shunting of blood. This shunt further reduces distal lung perfusion and compromises gas exchange, leading to intractable hypoxaemia and death. Focused studies on the regulation of IBA may lead to unique strategies that can attenuate the morbidity and mortality of patients who contracted SARS-CoV-2.

Csaba Galambos $\oplus^{1,2,3}$, Douglas Bush ${ }^{4}$ and Steven H. Abman $\oplus^{1,2,3}$

${ }^{1}$ Pediatric Heart Lung Center, Aurora, CO, USA. ${ }^{2}$ Children's Hospital Colorado, Aurora, CO, USA. ${ }^{3}$ University of Colorado Anschutz School of Medicine, Aurora, CO, USA. ${ }^{4}$ Icahn School of Medicine at Mount Sinai, New York, NY, USA.

Corresponding author: Csaba Galambos (csaba.galambos@childrenscolorado.org) 
Acknowledgements: The authors would like to acknowledge support from Fumiko Dekio and Rachel Brody from the Dept of Pathology at the Icahn School of Medicine at Mount Sinai.

Conflict of interest: None declared.

Support Statement: This work was supported by the National Heart, Lung, and Blood Institute (grant: NIH RO1 \# 2RO1HL06702-13A1). Funding information for this article has been deposited with the Crossref Funder Registry.

\section{References}

1 Gattinoni L, Coppola S, Cressoni M, et al. COVID-19 does not lead to a 'typical' acute respiratory distress syndrome. Am J Respir Crit Care Med 2020; 201: 1299-1300.

2 Porfidia A, Pola R. Venous thromboembolism in COVID-19 patients. J Thromb Haemost 2020; 18: 1516-1517.

3 Patel BV, Arachchillage DJ, Ridge CA, et al. Pulmonary angiopathy in severe CoVID-19: Physiologic, imaging, and hematologic observations. Am J Respir Crit Care Med 2020; 202: 690-699.

4 Ackermann M, Verleden SE, Kuehnel M, et al. Pulmonary vascular endothelialitis, thrombosis, and angiogenesis in Covid-19. N Engl J Med 2020; 383: 120-128.

5 Reynolds AS, Lee AG, Renz J, et al. Pulmonary vascular dilatation detected by automated transcranial doppler in COVID-19 pneumonia. Am J Respir Crit Care Med 2020; 202: 1037-1039.

6 Galambos C, Sims-Lucas S, Abman SH. Histologic evidence of intrapulmonary anastomoses by three-dimensional reconstruction in severe bronchopulmonary dysplasia. Ann Am Thorac Soc 2013; 10: 474-481.

7 Galambos C, Sims-Lucas S, Abman SH. Three-dimensional reconstruction identifies misaligned pulmonary veins as intrapulmonary shunt vessels in alveolar capillary dysplasia. J Pediatr 2014; 164: 192-195.

8 Ali N, Abman SH, Galambos C. Histologic evidence of intrapulmonary bronchopulmonary anastomotic pathways in neonates with meconium aspiration syndrome. J Pediatr 2015; 167: 1445-1447.

9 Acker SN, Mandell EW, Sims-Lucas S, et al. Histologic identification of prominent intrapulmonary anastomotic vessels in severe congenital diaphragmatic hernia. J Pediatr 2015; 166: 178-183.

10 Galambos C, Sims-Lucas S, Abman SH, et al. Intrapulmonary bronchopulmonary anastomoses and plexiform lesions in idiopathic pulmonary arterial hypertension. Am J Respir Crit Care Med 2016; 193: 574-576.

11 Galambos C, Bates ML, Bush D, et al. Prominent bronchopulmonary vascular anastomoses in fatal childhood asthma. Ann Am Thorac Soc 2018; 15: 1359-1362.

12 Bush D, Abman SH, Galambos C. Prominent intrapulmonary bronchopulmonary anastomoses and abnormal lung development in infants and children with down syndrome. J Pediatr 2017; 180: 156-162 e1.

13 Dorfmuller P, Gunther S, Ghigna MR, et al. Microvascular disease in chronic thromboembolic pulmonary hypertension: a role for pulmonary veins and systemic vasculature. Eur Respir J 2014; 44: 1275-1288.

14 McMullan DM, Hanley FL, Cohen GA, et al. Pulmonary arteriovenous shunting in the normal fetal lung. J Am Coll Cardiol 2004; 44: 1497-1500.

15 Eldridge MW, Dempsey JA, Haverkamp HC, et al. Exercise-induced intrapulmonary arteriovenous shunting in healthy humans. J Appl Physiol 2004; 97: 797-805.

16 Lovering AT, Romer LM, Haverkamp HC, et al. Intrapulmonary shunting and pulmonary gas exchange during normoxic and hypoxic exercise in healthy humans. J Appl Physiol 2008; 104: 1418-1425.

17 Laurie SS, Elliott JE, Goodman RD, et al. Catecholamine-induced opening of intrapulmonary arteriovenous anastomoses in healthy humans at rest. J Appl Physiol 2012; 113: 1213-1222.

18 Lovering AT, Elliott JE, Beasley KM, et al. Pulmonary pathways and mechanisms regulating transpulmonary shunting into the general circulation: an update. Injury 2010; 41: Suppl. 2, S16-S23.

19 Andrey P, Maurin Y. Free-D: an integrated environment for three-dimensional reconstruction from serial sections. J Neurosci Methods 2005; 145: 233-244.

20 Bush D, Deterding R, Weinman J, et al. Hypoxemia in lipoid pneumonia: role of intrapulmonary bronchopulmonary anastomoses. Am J Respir Crit Care Med 2017; 195: 1531-1532.

21 Marchand P, Gilroy JC, Wilson VH. An anatomical study of the bronchial vascular system and its variations in disease. Thorax 1950; 5: 207-221. 